\title{
Non-septic Hip Instability in the Chronic Spinal Cord Injury Patient
}

\author{
R. A. Baird, M.D., ${ }^{1.2}$ M. J. DeBenedetti, M.D., ${ }^{2}$ I. Eltorai, M.D. ${ }^{3}$ \\ From the ${ }^{1} S T A M P$ Program; ${ }^{2}$ The Section of Orthopaedic Surgery; and ${ }^{3}$ The \\ Spinal Cord Injury Service of the Long Beach Veteran's Administration Medical \\ Center, 5901 East Seventh Street, Long Beach, California 90822, U.S.A.
}

\section{Introduction}

The most common causes of subluxation and dislocation of the hip joint in the chronic spinal cord injury (SCI) patient are intra-articular and periarticular infections of the hip, and surgery about the hip for soft tissue and bone debridement. Hip instability without antecedent infection or surgery is uncommon. To date, the etiology, natural history, and sequelae of non-septic subluxation and dislocation of the hip joint in SCI patients have not been reported. Recent experience with a potentially surgically reducible hip joint subluxation in a young man with an incomplete $T-4$ paraplegia led us to examine our patient population in an effort to determine the natural course and sequelae of non-septic hip instability in the chronic spinal cord injury patient.

\section{Materials and methods}

The radiographs of patients treated by the Spinal Cord Injury Service at the Long Beach Veterans Administration Medical Center were reviewed for evidence of hip joint instability. The charts of patients whose radiographs demonstrated hip subluxation or dislocation were reviewed for documentation of sepsis, possible sepsis, or previous surgery on the affected hip as causative factors of the hip instability. Patients without a history of sepsis or surgery in the region of the hip prior to the onset of instability were contacted and were requested to submit to a personal interview and physical examination. Personal interview investigated the etiology of the hip instability, and the affect of hip instability upon function, ambulation, use of a standing frame, sitting balance and sitting tolerance, the presence of pain, and the occurrence of decubitus ulcers. Physical examination was performed evaluating hip range of motion, presence and degree of scoliosis, and pelvic obliquity in the sitting position. When necessary, radiographs were performed to document the progression of hip instability, femoral head resorption, and scoliosis. Radiographs of the spine were performed with the patient in the sitting position. 
Table 1 Pain Grading Scale

$0-N O N E-$ No noticeable pain

1-MINIMAL-Infrequent pain not requiring medication or treatment; not affecting function or lifestyle.

2-MILD-Intermittent or constant pain requiring use of non-narcotic analgesics; not affecting function or lifestyle.

3-MODERATE-Intermittent or constant pain requiring narcotic medication or other treatment (TENS; biofeedback, etc.); not affecting function or lifestyle.

$4-S E V E R E$ - Intermittent or constant pain, unrelieved by oral doses of narcotic analgesics or other usual forms of treatment (TENS; biofeedback, etc.); causing mild to moderate limitation of function or lifestyle.

5-INCAPACIT ATING-Constant pain, unrelieved by usual forms of treatment; limiting function and lifestyle to a significant degree.

Table 2 Levels of Sitting Tolerance

1. Unrestricted ability to sit in cushioned wheelchair without pain or skin problems.

2. Mild restriction in ability to sit. Pain or aching on sitting may be present. Able to sit longer than eight hours.

3. Inability to sit longer than eight hours secondary to pain or skin problems.

4. Inability to sit more than two hours.

5. Inability to sit for more than one-half hour.

6. Inability to sit.

None-no change in level

Grading of Diminution in Sitting Tolerance

Mild-change of one level

Moderate-change of two levels

Severe-change of three levels

Table 3 Levels of Sitting Balance

1. Balanced sitting posture-No external support or repositioning necessary.

2. Mild imbalance-Uneven cushion wear may be noted. Occasional repositioning for balance necessary. No external support needed.

3. Moderate imbalance-Frequent repositioning for balance necessary. Occasional need for external support (arms, brace or jacket, armrests).

4. Significant imbalance-Frequent repositioning necessary. Frequent need for external support.

5. Marked imbalance-Unable to sit unsupported.

None-no change in level

Grading of Diminution in Sitting Balance

Mild-change of one level

Moderate-change of two levels

Severe-change of three levels

Pain was graded on a scale from Grade 0 (none) to Grade 5 (incapacitating) as outlined in Table 1. Sitting tolerance was graded on a six-level scale with 
Level 1 being the unrestricted ability to sit in a cushioned wheelchair without pain or skin problems, and Level 6 being the inability to sit (Table 2). The ability to sit for a period of eight hours, the normal workday in the United States, was the dividing point between sitting tolerance Level 2 and Level 3. A change of one level was defined as a mild diminution in sitting tolerance, a change of two levels was defined as moderate, and a change of three or more levels was defined as severe diminution in sitting tolerance. A five-level scale was used to evaluate sitting balance. (Table 3 ). Diminution in sitting balance was determined in a fashion similar to that for sitting tolerance.

\section{Results}

Twenty-four patients were found to have sustained non-septic subluxation or dislocation of the hip between 1965 and 1983. Seven patients were deceased. Of the remaining 17 patients, we were able to contact 10, all of whom consented to interview and physical examination. Eight patients were found to have a unilateral hip dislocation, one patient had bilateral hip dislocations, and one patient had a unilateral hip subluxation. Thus, there were 11 involved hips in 10 patients. All 10 patients were male.

Two of the 10 patients were quadriplegic with neurologic levels between C-3 and C-6, and eight were paraplegic with levels between T-4 and T-10. Six had incomplete spinal cord lesions and four had complete lesions. The average age at time of spinal cord injury was 27 years with a range of 21 years to 40 years. The cause of the spinal cord injury was a motor vehicle accident in five and a gunshot wound in five. Eight of the involved hips were on the left side and three were on the right.

The cause of instability was unknown in six hips, attributed by the patient to muscle spasms in three, attributed to a fall from a wheelchair during transfers in one, and attributed to the use of standing frame in one. The interval between spinal cord injury and the time that hip dislocation was recognised by the patient's treating physician (in nine patients with 10 dislocated hips) averaged eight years with a range of one and one-half years to 34 years three months. At the time hip instability was recognised, patient's age ranged from 27 to 55 years, with a mean age of 36 . The mean length of follow-up was five years eight months (range 8 months to 18 years six months).

\section{Symptoms}

Three patients, two with incomplete lesions and one with a complete lesion, denied exeriencing any symptoms in their hip during the time period in which the hip instability occurred. Two patients with incomplete lesions and one patient with a complete lesion complained of increased muscle spasms in the affected extremity during this time period. Two patients with complete lesions and one patient with an incomplete lesion complained of hip discomfort or aching pain. Physical signs included thigh swelling in one patient, the sensation of the hip joint going in and out of place in one patient, and shortening of the limb in one patient. 
Five patients complained of hip pain present at the time of follow-up interview. Of these five patients, three described their pain as a dull, mild ache (Grade 1). One patient, with incomplete C-3 quadriplegia, complained of severe (Grade 4) hip pain. However, this patient's pain could be markedly diminished with intramuscular saline injections. A second patient, with incomplete T-10 paraplegia, complained of severe (Grade 4) pain, but required no treatment for pain relief. Five patients had no pain at time of follow-up.

\section{Ambulatory and Sitting Function}

No patient in this series was an ambulator prior to the onset of hip instability. However, four of the 10 patients used a standing frame. The time interval between spinal cord injury and the recognition of hip instability in these patients ranged from nine months to six years (mean three years). At the time of follow-up, none of these patients had resumed use of the standing frame.

Prior to the onset of hip instability, levels of sitting tolerance in this patient population ranged from Level 1 (unrestricted ability to sit) to Level 2 (mild restriction in the ability to sit but with the ability to sit longer than eight hours). At follow-up, the patients' level of sitting tolerance ranged between Level 1 and Level 4 (inability to sit more than two hours). Six of the 10 patients had no diminution in their ability to sit secondary to their hip instability. Two patients experienced a mild (one level) diminution in sitting tolerance, one from Level 1 to Level 2, and one from Level 2 to Level 3. Two patients experienced a moderate (two level) diminution in sitting tolerance secondary to their hip instability, one from Level 1 to Level 3, and one from Level 2 to Level 4. These changes in sitting tolerance correlated with the patient's subjective evaluation of their ability to sit.

Prior to hip instability, levels of sitting balance ranged from Level 1 (balanced sitting posture) to Level 5 (marked imbalance, with inability to sit unsupported). Only two patients had a diminution in sitting balance secondary to their hip instability. One patient experienced moderate diminution (Level 1 to Level 3), and one patient experienced severe diminution (Level 1 to Level 4). These patients' subjective evaluations of their sitting balance correlated well with the sitting balance scale. Two other patients felt that their sitting balance was diminished, one to a mild degree and one to a moderate degree. Using the sitting balance scale, however, neither patient experienced a change in level of sitting balance.

\section{Decubitus Ulcer Formation}

All 10 patients had developed decubitus ulcers at some time between their spinal cord injury and follow-up evaluation for this study. During the interval between spinal cord injury and follow-up interview, the 10 patients sustained a total of 27 decubitus ulcers. Nine of these ulcers developed prior to the onset of hip instability, five during the year of onset of hip instability, and 13 subsequent to the development of hip instability. Prior to hip instability, the incidence of decubitus ulcers (nine ulcers in 107 patient-years) was one ulcer per 11.9 
Table 4 Location of Decubitus Ulcers

\begin{tabular}{lcccc}
\hline & Ipsilateral & Sacral & Contralateral & Total \\
Prior to Hip Instability & $4\left(45^{\circ}{ }_{0}\right)$ & $3\left(33^{\prime}{ }_{0}\right)$ & $2\left(22^{\circ}{ }_{0}\right)$ & $9\left(100^{\prime \prime}{ }_{0}\right)$ \\
After Onset of Hip Instability & $2\left(15^{\circ}{ }_{0}\right)$ & $1\left(8^{\prime \prime}\right)$ & $10\left(77^{\prime \prime}\right)$ & $13\left(100^{\prime \prime}\right)$ \\
\hline
\end{tabular}

$\star$ To the unstable hip.

patient-years. Following hip instability, the incidence of decubitus ulcers (13 ulcers in 44 patient-years) was one ulcer per 3.4 patient-years.

Prior to the onset of hip instability there was a slight preponderance of decubitus ulcers (Table 4) on the side ipsilateral to the subsequently unstable hip (four ulcers), compared to the contralateral side (two ulcers), and the sacrum (three ulcers). After hip instability this pattern reversed, with a marked preponderance of decubitus ulcers on the side opposite the hip instability (10 ulcers) as compared to the ipsilateral side (two ulcers) and the sacrum (one ulcer).

\section{Patient Complaints}

At follow-up, one patient had no complaints referrable to his dislocated hip. Five patients stated that the greatest problem their dislocated hip caused them was an increase in muscle spasms in the affected leg. Two patients complained of an increase in the incidence of decubitus ulcers, one complained of severe pain; and one complained of difficulty lying prone.

\section{Hip Motion}

Seven patients underwent examination of hip range of motion at follow-up. Of these seven, one patient had undergone bilateral femoral head and neck resection, one patient had bilateral hip dislocations, and one patient had a contralateral femoral head and neck resection subsequent to recognition of their hip dislocation and prior to follow-up examination. A comparison of range of motion between the unstable hip and the stable hip could therefore be performed at follow-up examination in only four patients who had unilateral hip dislocation without subsequent surgery on either the stable or unstable hip. In these four patients, there was a moderate reduction in hip abduction (mean $13^{\circ}$ ), a mild reduction in flexion (mean $12^{\circ}$ ), and no reduction in hip extension. There was no significant difference between the stable hip and the unstable hip with regard to internal or external rotation in either the flexed or extended position.

\section{Scoliosis}

Eight of the 10 patients demonstrated scoliosis on follow-up radiographic examination of the spine. The location of the curves were thoracic in one patient, thoracolumbar in four patients, lumbar in two patients, and combined thoracic and lumbar in one patient. In five of the eight patients with scoliosis, equivalent radiographs before and af ter hip instability permitted the evaluation of possible scoliosis progression. One of the five patients demonstrated no progression, 
three patients demonstrated mild curve progression $\left(7^{\circ}, 9^{\circ}\right.$, and $\left.9^{\circ}\right)$, and one patient demonstrated 12 degrees of curve regression.

\section{Pelvic Obliquity}

Six patients were examined in the sitting position for the presence of pelvic obliquity. Five patients were considered to have mild pelvic obliquity, with moderate obliquity in one. The patient with a moderate degree of pelvic obliquity had an associated thorcolumbar C-type scoliosis of less than 10 degrees in magnitude. The unstable hip was ipsilateral to the high side of the pelvis in all six patients in this series who had pelvic obliquity.

\section{Discussion}

In the chronic spinal cord injury patient, hip instability is most commonly secondary to pyarthrosis of the hip joint which erodes the bony structures of the hip joint and distends and stretches the peri-articular soft tissues. Hip subluxation and dislocation are also seen following soft tissue infections and surgical procedures about the hip, and osteomyelitis of the greater trochanter. Non-septic hip instability in the chronic spinal cord injury patient is uncommon.

In the non-spinal cord injury patient, one must consider trauma, congenital and developmental deformity, paralytic pelvic obliquity, and spasticity among the causes of non-septic hip instability. In this series of SCI patients, no patient sustained major trauma to his lower extremities during the time period in which the hip instability occurred. Additionally, no patient had radiographic evidence of congenital or developmental hip deformity. For these reasons, paralytic pelvic obliquity and spasticity appear to be the most likely causes of non-septic hip instability in this patient population.

Severe paralytic obliquity may cause hip dislocation on the high side of the pelvis (O'Brien, 1975) and could considerably be a cause of hip dislocation in the spinal cord injury patient. When significant pelvic obliquity is present, a loss of sitting balance is common, and pressure necrosis and ischial decubiti occur on the down side of the gluteal region. However, the data do not support this as a cause of hip instability in our patient population for several reasons. The pelvic obliquity seen in our patients at follow-up (mild in five, moderate in one) was not of the degree usually seen in cases of paralytic pelvic obliquity. The decubiti observed in our patient population prior to hip instability were more often ipsilateral than contralateral to the high side of the pelvis and the side of hip instability. This is opposite to the pattern of increased number of contralateral (down side) decubiti seen in patients with paralytic pelvic obliquity and hip dislocation. Lastly, prior to hip instability $60^{\circ}$ o of the patients in this series had balanced (Level 1) sitting posture requiring no external support or repositioning. This would be unlikely if the hip instability was caused by paralytic pelvic obliquity. Thus, we do not feel that paralytic pelvic obliquity was the cause of hip instability in our series.

The most probable cause of hip instability in the chronic SCI patient without sepsis in the region of the hip joint appears to be spasticity. This hypothesis is supported by the preponderance of patients with incomplete spinal cord injuries 
in this series, and the fact that two of the four patients with complete neurological lesions had greater than average levels of lower extremity spasticity. In contrast to patients with cerebral palsy, in whom spastic hip dislocation is usually associated with fixed flexion and adduction contractures, significant contractures were not seen in the patients reported in this series. Thus, although spasticity may be the dominant factor in the pathogenesis of non-septic hip dislocation in the spinal cord injury patient, other unknown factors may also play a part in this mechanical process.

Because the SCI patient's level of function may change over time, and because of the variable functional effects which were noted in this study, few generalisations can be made about the effect of hip instability upon function. All four patients who were standing frame users prior to their onset of hip instability ceased using the frame shortly thereafter. However, as many spinal cord injury patients eventually discontinue using a standing frame at home, it remains possible that these patients would have discontinued use of the standing frame even without the presence of hip instability. While hip instability appeared to have little or no effect upon sitting tolerance and sitting balance in seven of the 10 patients, three patients complained of moderate to severe diminution in these factors. Two of these three patients noted a significant increase in lower extremity muscle spasm after hip dislocation. This increase in spasticity may relate to their perceived diminution in sitting tolerance and balance.

The most significant effects of the hip instability upon this patient population were an increase in muscle spasms, experienced by $50^{\circ}$ of the patients, and a change in the frequency and pattern of decubitus ulcers. The frequency of decubitus ulcer formation increased more than threefold, from one ulcer per 11.4 patient-years to one ulcer per 3.4 patient-years. The distribution of the ulcers also changed, from $22^{\circ}{ }_{0}$ of the ulcers contralateral to the unstable hip prior to instability, to $77^{\circ}$ o contralateral to the affected hip after instability. It would appear from these data that hip instability causes a shift in the pattern of sitting pressure from one which has a relatively even pressure distribution, to one which has an uneven distribution, with greater pressures under the buttock contralateral to the unstable hip.

Because hip instability may cause significant diminution in sitting tolerance and balance in certain patients, we feel that hip instability should be prevented if at all possible. Leg spreaders, passive stretching, obturator neurectomy, and flexor/adductor releases may be necessary in patients with either hip flexor/ adductor spasticity or contractures. Once instability has developed, the level of symptomatology is the best guide to treatment. Proximal femoral resection may be successful in reducing the pain associated with an unstable hip in patients with an incomplete spinal cord injury. What effect such treatment has upon the incidence of post-instability decubitus ulcer formation is uncertain. Studies are now being undertaken to evaluate the effects of hip instability and proximal femoral resection upon sitting pressures in the chronic SCI patient.

\section{References}

Bonnett C, Brown JC, Perry J, et al. 1975 Evolution of Treatment of Paralytic Scoliosis at Rancho Los Amigos Hospital. Journal of Bone and Joint Surgery 57-A(2):206-215. 
O'Brien JP, Dwyer AP, Hodgson AR 1975 Paralytic Pelvic Obliquity. Journal of Bone and Joint Surgery 57-A(5):626-631.

SRIRAM K, Bobechko WP, Hall JE 1972 Surgical Management of Spinal Deformities in Spina Bifida. Journal of Bone and Joint Surgery 54-B(4):666-676. 